\title{
Optimal duration of anticoagulant therapy in patients with venous thromboembolism
}

\author{
Gualtiero Palareti \\ Cardiovascular Diseases, University of Bologna; President of Arianna Anticoagulazione Foundation, Bologna, Italy
}

\begin{abstract}
Venous thromboembolism, a frequent and severe disease, has clinically important early and late complications and a strong tendency to recur. Anticoagulant therapy is the mainstay of treatment, performed by immediate administration of: i) parenteral anticoagulants followed by vitamin $\mathrm{K}$ antagonists, either dabigatran or edoxaban, two direct oral anticoagulants (DOACs); or ii) direct rivaroxaban or apixaban, two DOACs that can be used as single-drug approach. Treatment should last no less than 3 months in all patients though how long it should last thereafter is a more complex issue. The risk of recurrence results from several event- or patient-associated factors. Some patients have low risk and may be treated for 3 to 6 months only. Others (the majority) have a high risk of recurrence (approximately 50\% in 10 years). Unfortunately, the protective effect of anticoagulation against recurrence is present only during treatment and is lost when therapy is stopped. For this reason, international guidelines recommend that there is no pre-definite period of anticoagulation (e.g. 1 or 2 years, and so on) in patients at high risk and suggest instead indefinite (extended) anticoagulation, provided there is no high risk of bleeding. When the decision is difficult, adjunctive criteria may be adopted, such as male sex and abnormal D-dimer assessed after anticoagulation is stopped, to identify patients at high risk who need indefinite therapy. The use of DOACs, especially at lower doses with a lower risk of bleeding, may make indefinite anticoagulation for patients easier.
\end{abstract}

\section{Introduction}

Venous thromboembolism (VTE), encompassing deep vein thrombosis (DVT) of lower limbs and/or pulmonary embolism (PE), is a severe, potentially lethal disease. Its incidence is high, affecting 1 to 2 out of 1000 persons per year in developed countries, ${ }^{1-3}$ and represents the third most common cardiovascular disease, after myocardial infarction and stroke. ${ }^{4}$ VTE is an acute disease that may have clinically important

Correspondence: Gualtiero Palareti, Cardiovascular Diseases, University of Bologna; President of Arianna Anticoagulazione Foundation, via Paolo Fabbri 1/3, 40138 Bologna, Italy. Fax: +39.051.343604

E-mail: gualtiero.palareti@unibo.it

Key words: Venous thromboembolism; anticoagulation; duration; D-dimer.

Conflict of interest: the author declares no conflict of interest.

Received for publication: 9 August 2018.

Accepted for publication: 22 August 2018.

This work is licensed under a Creative Commons Attribution NonCommercial 4.0 License (CC BY-NC 4.0).

(C) Copyright G. Palareti, 2018

Licensee PAGEPress, Italy

Italian Journal of Medicine 2018; 12:235-244

doi:10.4081/itjm.2018.1077 outcomes early and late after initial presentation. All patients with acute VTE need immediate active anticoagulant therapy. This treatment may require administration of: i) parenteral drugs (heparin or derivatives) followed by vitamin $\mathrm{K}$ antagonists (VKAs) or by the direct oral anticoagulants (DOACs) dabigatran and edoxaban; ${ }^{5,6}$ or ii) oral administration of the DOACs apixaban or rivaroxaban, drugs that may be used as a first-alone drug in the first days of treatment and in the following months, at the appropriate dosages. ${ }^{7,8}$

VTE may also have late, clinically important consequences. Post-thrombotic syndrome (PTS), a condition that greatly affects the morbidity and quality of life of patients and is associated with high social costs, is a late outcome of DVT. PTS, particularly frequent after recurrent DVT in the same leg of the first event, was detected in mild, moderate or severe form in $30 \%$, $10 \%$ and $3 \%$ of patients, respectively, two years after first DVT. ${ }^{9}$ Another important late complication is chronic thromboembolic pulmonary hypertension, detected in as many as $3.8 \%$ of patients two years after an acute episode of PE. ${ }^{10}$

\section{Anticoagulation to treat acute venous thromboembolism and to avoid recurrences}

Immediate anticoagulation is very effective against DVT or PE extension and new early episodes in the acute phase of the disease (sentence not clear, please rephrase), as well as against VTE recurrence in post- 
acute phase; this is true when either $\mathrm{VKAs}^{11}$ or DOACs $^{5-8}$ are used. VTE has, however, a strong tendency to recur. Though recurrent VTE episodes or extension of the disease may occur in some patients even in the presence of adequate therapy $(\approx 4 \%$ after a DVT),${ }^{12,13}$ the risk of recurrence increases sharply after anticoagulation is stopped. This is why some authors claim VTE is a chronic disease. ${ }^{14}$

Clinical studies have investigated the effects of different anticoagulant treatment times in subjects after VTE event, and found that three-month treatment gives better results than a shorter period and achieves a similar risk of recurrence after anticoagulation cessation as a longer course of treatment. ${ }^{15}$ Furthermore, it was shown that whatever the duration of the anticoagulant treatment, its benefit faded after anticoagulation was stopped and the risk of recurrence increased again. ${ }^{16-19}$ Based on these results, the recent American College of Chest Physician (ACCP) guidelines recommend that all patients with acute VTE should receive no less than 3 months of anticoagulation, a period considered necessary to cover the initial and maintenance phases of VTE. A longer but definite time-period of anticoagulation (e.g. 1 year, 2 years, etc.) is not recommended, since the risk of recurrence increases again after therapy is stopped. ${ }^{20}$ The mentioned guide- lines suggest, therefore, that patients at high risk of recurrent VTE and non-high risk of bleeding should be evaluated for indefinite anticoagulant treatment, i.e. no pre-established limited duration of treatment, with periodical clinical control of patient's conditions.

\section{The clinical risk associated with recurrent venous thromboembolism and risk factors for recurrences}

The patients' clinical conditions significantly worsen after recurrent events. A recurrent DVT in the same leg strongly increases the risk of developing postthrombotic syndrome [heart rate 6.4; confidence interval (CI) 3.1-13.3] and it is associated with a reduction in survival..$^{21,22}$ Recurrent PE is the strongest risk factor for chronic pulmonary hypertension ${ }^{23}$ with the risk of recurrence falling over time after the first VTE. A recent systematic review found a $0.4 \%$ (CI $0.3-0.6)$ rate of fatal recurrent VTE, with a case-fatality rate of $11.3 \%$ (CI 8.0-15.2) during the first 3 months of anticoagulant therapy; after the first 3 months the rate of fatal recurrence was $0.3 \%$ patient-years (CI $0.1-0.4)$, with a case fatality rate reduced to $3.6 \%$ (CI 1.9-5.7). ${ }^{24}$

The risk factors for VTE recurrence may be related

Table 1. Risk factors for venous thromboembolism recurrence.

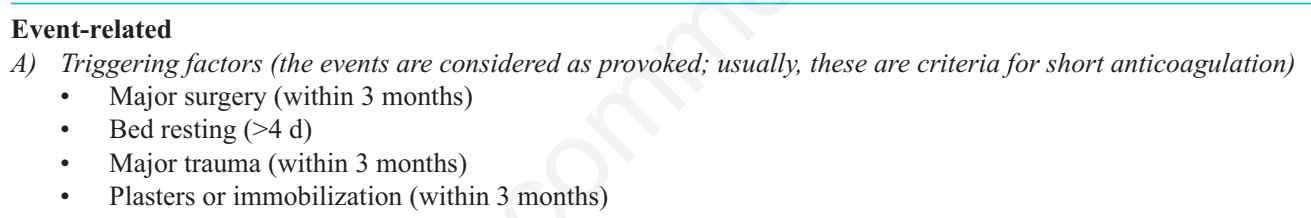

B) Weak factors (the associated risk and usefulness of extended anticoagulation are still uncertain)

- Minor general, laparoscopic, or arthroscopic surgery

- Long travel time

- Minor trauma, leg injury, reduced mobility

- Hospitalization in a medical ward

- Pregnancy or puerperium

Patient-related

A) Major factors (usually considered as criteria for extended anticoagulation)

- More than 1 documented VTE episode (proximal DVT and/or PE)

- Previous PE event presenting with shock or life-threatening prolonged hypotension

- Active cancer or hematologic disease

- Antithrombin deficiency or other major inherited thrombophilic alterations

- Antiphospholipid antibody syndrome (Sydney criteria)

- Severe cardiorespiratory insufficiency (NYHA 3 or 4)

- Active inflammatory bowel disease

B) Other factors

- Males

- Young age

- Minor thrombophilic alterations

- High BMI

- Persistence of residual vein thrombi

- Signs of hypercoagulability (increased D-dimer, high plasma F, VIII levels, thrombin generation assays)

- Contraceptive or replacement hormonal therapy

- $\quad$ Reduced mobility (not complete immobilization)

VTE, venous thromboembolism; DVT, deep vein thrombosis; PE, pulmonary embolism; NYHA, New York Heart Association; BMI, body mass index. 
to the nature and type of the index event, and to the characteristics of the patient (Table 1). Patients whose VTE occurred without any strong VTE risk factor (unprovoked VTE) benefit from extended anticoagulation therapy, whereas those with VTE associated with a triggering and removed major risk factor do not. Furthermore, a recent study showed that some patients with VTE associated to minor persistent or transient risk factors may also benefit from extended anticoagulation therapy. ${ }^{25}$

\section{Timing and criteria to decide the duration of anticoagulation in individual venous thromboembolism patients}

\section{When to tackle the decision}

Some clinical conditions which clearly point to short or extended anticoagulation may be present at the moment of VTE diagnosis; the treating clinicians can therefore inform the patients about the expected duration of anticoagulant therapy at the moment of diagnosis. In most cases, however, the issue of anticoagulation duration is assessed only after 3 to 6 months of treatment. Many factors may affect the risk of recurrent VTE events and more risk factors can be present in a single patient. Each patient should, therefore, be evaluated paying attention to all possible influencing factors and also to his/her preference (Table 2).

\section{Candidates for a short anticoagulation period}

Candidates for a short anticoagulation period (usually lasting 3 to 6 months) are the following:
- Patients whose event was limited to the deep calf veins, without involving proximal veins (isolated distal DVT, IDDVT), have a lower risk of recurrence $^{16,26}$ than proximal DVT. Though the longterm risk of recurrence after IDDVT is still uncertain, ${ }^{27}$ a limited period of anticoagulation (3 months) is recommended, unless other risk factors (such as cancer or major thrombophilic alterations) are present.

- Patients whose first VTE event was associated with a temporary (and removed) triggering factor (such as surgery, trauma, plasters, immobility, prolonged bed resting, etc.) have a low risk of recurrence, with surgery associated with the lowest risk of recurrence. ${ }^{28}$ These patients had a so-called provoked VTE event and deserve a short treatment period.

- Patients who have a high risk of bleeding during anticoagulation are certainly candidates for short anticoagulation. ${ }^{20}$

\section{Candidates for extended anticoagulation}

Candidates for extended anticoagulation (for an indefinite period), due to the presence of major and persisting risk factors (conditions associated with a very high risk of recurrence) are the following:

- Patients who had more than one unprovoked VTE episode, have a very high risk of recurrence and deserve indefinite anticoagulation. ${ }^{29}$

- The presence of active cancer is one of the most important persistent factors associated with VTE recurrence, either after anticoagulation is stopped ${ }^{21}$ or even during treatment; ${ }^{30,31}$ the risk further increases during concurrent chemotherapy. ${ }^{32}$

- Carriers of major inherited thrombophilic alter-

Table 2. Patient categories, related risk of venous thromboembolism recurrence and suggested duration of anticoagulation.

\begin{tabular}{|c|c|c|c|}
\hline Patient categories & Clinical conditions & Risk of recurrence & $\begin{array}{l}\text { Suggested duration } \\
\text { of anticoagulation }\end{array}$ \\
\hline All patients with a VTE event & All possible conditions & $\begin{array}{l}\text { Whatever the risk of } \\
\text { recurrence and of bleeding }\end{array}$ & Not $<3$ months \\
\hline $\begin{array}{l}\text { Patients with a } 1^{\text {st }} \text { VTE event } \\
\text { associated with a trigger } \\
\text { (and removed) factor }\end{array}$ & $\begin{array}{l}\text { Surgery, trauma, plasters, immobility, } \\
\text { prolonged bed resting, etc. }\end{array}$ & Low & 3-6 months \\
\hline Patients at high risk of bleeding & $\begin{array}{l}\text { According to the ACCP score } \\
\text { (or other criteria) }\end{array}$ & $\begin{array}{l}\text { Whatever the risk of } \\
\text { recurrence }\end{array}$ & 3 months \\
\hline Patients at high risk of recurrence & $\begin{array}{l}>1 \text { unprovoked VTE episodes, active } \\
\text { cancer, major thrombophilic alterations, } \\
\text { APS, presentation as hemodynamic PE }\end{array}$ & High & Indefinite \\
\hline $\begin{array}{l}\text { Patients after a } 1^{\text {st }} \text { unprovoked } \\
\text { VTE, (or associated with a weak } \\
\text { risk factor) }\end{array}$ & $\begin{array}{l}\text { Idiopathic event; and/or males, young age, } \\
\text { presence of RVT, obesity, high clotting } \\
\text { factor levels, inherited thrombophilia, } \\
\text { long distance travel, inflammatory bowel } \\
\text { disease (others?) }\end{array}$ & High (or probably high) & $\begin{array}{l}\text { Indefinite*; } \\
\text { Alternatively: D-dimer-based } \\
\text { procedure to identify patients } \\
\text { at high or non-high risk of } \\
\text { recurrence }\end{array}$ \\
\hline
\end{tabular}

VTE, venous thromboembolism; ACCP, American College of Chest Physician; APS, antiphospholipid syndrome; PE, pulmonary embolism; RVT, residual vein thrombosis. *Indefinite = without a pre-definite stopping date, but with periodical checks to assess the patient conditions and the current balance between benefits and risks of extending anticoagulation. 
ations or antiphospholipid syndrome. Though rare, these alterations, that were shown to be associated with higher risk of recurrent thrombotic events, include: deficiency of physiological anticoagulants (antithrombin, protein $\mathrm{C}$ and protein S), ${ }^{21}$ presence of homozygous or double heterozygous factor $\mathrm{V}$ Leiden and prothrombin G20210A mutations, ${ }^{33,34}$ and antiphospholipid syndrome. ${ }^{35}$ In contrast, the presence of the very frequent heterozygous factor $\mathrm{V}$ Leiden or prothrombin $620210 \mathrm{~A}$ mutations do not seem to justify indefinite anticoagulation.

- Patients whose index event was a PE presenting with shock or life-threatening prolonged hypotension. This is justified by studies reporting that patients presenting with $\mathrm{PE}$ are more likely to suffer from a new PE by way of recurrence than patients presenting with DVT, thus exposing them to higher clinical risk. ${ }^{26,36} \mathrm{In}$ a retrospective study, ${ }^{37}$ VTE recurrence was in $70 \%$ of cases a new PE episode in patients presenting with PE, but only $15 \%$ in patients with DVT. Furthermore, the increased clinical risk associated with recurrent $\mathrm{PE}$ episodes was demonstrated by Douketis et al. ${ }^{38}$ who found a higher rate of recurrent fatal PE in patients with a first VTE presenting as PE than in those with DVT ( $1.2 \%$ vs $0.3 \%$, respectively).

\section{Patients whose venous thromboembolism was associated with non-surgical risk factors (probably associated with increased risk)}

Several VTE-associated risk factors have been identified and discussed ${ }^{39}$ though clinical research is still needed to conclude whether or not their presence needs extended anticoagulation. Currently their presence/absence is not sufficient to decide the duration of anticoagulation in single patients:

- The persistence of residual vein thrombosis (RVT) in DVT-involved deep veins of lower limbs was found to be associated with the risk of recurrent DVT in some ${ }^{40-43}$ though not all studies. ${ }^{44-46}$ Two randomized studies showed that persistent RVT was a good criterion for determining the duration of anticoagulation. ${ }^{47,48} \mathrm{~A}$ recent systematic review concluded that after a first unprovoked DVT, RVT is a weak overall predictor of recurrent VTE, with a stronger association if RVT is detected early after thrombosis. ${ }^{49}$ In the DULCIS study (D-dimer and ULtrasonography in Combination Italian Study) ${ }^{50}$ patients with RVT at screening received one year of anticoagulation and after this period no difference was recorded in the rates of recurrent events in relation to the presence/absence of RVT in patients who stopped anticoagulation.

- Men are exposed to higher risk than females. ${ }^{51,52}$ A recent study showed that men had a 2.2-fold higher risk of recurrent events than women, a difference that was present only after an unprovoked VTE and disappeared when the first event was provoked. ${ }^{53}$

- The effect of increasing age on the risk of recurrence is still uncertain, since an increased risk has been detected only in some, ${ }^{22,32,54}$ though not all studies. ${ }^{55-57}$

- Being overweight and obese were found to be associated with a higher risk of recurrence..$^{58}$

- Increased levels of some blood clotting factors were associated with the risk of a first and also of recurrent VTE; this was shown for factor VIII ${ }^{59,60}$ and factor IX. ${ }^{61}$

- Some inherited thrombophilic alterations, such as the heterozygous presence of factor $\mathrm{V}$ Leiden or of prothrombin $\mathrm{G} 20210 \mathrm{~A}$ mutations, highly frequent in general Caucasian population, are risk factors for VTE but do not confer a higher risk of recurrence. ${ }^{62,63} \mathrm{~A}$ meta-analysis of prospective studies demonstrated only a slightly increased risk of recurrent VTE in patients who had a heterozygous FV factor V Leiden (relative risk 1.4, 95\% CI 1.1-1.8) or prothrombin G20210A mutation (relative risk $1.7,95 \%$ CI 1.3-2.3) ${ }^{64}$ In these patients, accurate antithrombotic prophylaxis in all conditions associated with increased thrombotic risk seems to be adequate.

- Recent guidelines ${ }^{65}$ indicate that long distance travel (usually considered at least $4 \mathrm{~h}$ ) is also a weak risk factor, with a risk that is higher in individuals with pre-existing risk factors for VTE.

- Patients with inflammatory bowel disease had a higher risk of recurrence according to a recent study. ${ }^{66}$

In conclusion, while the possible presence of these factors should be assessed, their presence in most cases is not enough to decide between short or extended anticoagulation.

\section{Absence of triggering factors (unprovoked events)}

Between $25 \%$ and $50 \%$ of all patients with first VTE had the event in the absence of major risk factors (so called unprovoked, or idiopathic events). ${ }^{7,67,68}$ In general, this category of patients is considered at high risk of recurrence and the recent AT 10 ACCP guidelines suggest giving extended anticoagulation to all subjects, provided they are not at high risk of bleeding. ${ }^{20}$ However, a better risk-stratification among this large group of unprovoked patients seems appropriate and has also been recommended ${ }^{69} \mathrm{In}$ this regard, the above-mentioned guidelines suggest treating physicians take into account other characteristics (such as male sex and abnormal D-dimer levels) in case of doubt about the duration of treatment. ${ }^{20}$ 


\section{The role of D-dimer testing to assess individual risk of venous thromboembolism (VTE) recurrence in unprovoked VTE patients}

Plasma D-dimer levels are considered an indirect marker of coagulation activation. Besides the well-established use of D-dimer assay for the diagnostic procedure in symptomatic outpatients with suspected acute VTE, the test has also been proposed to assess individual risk of recurrent VTE. A prospective, inception-cohort study, for the first time showed a significantly higher hazard ratio for recurrence in subjects with abnormal versus normal D-dimer at 3 months $(2.45 ; 95 \%$ CI $1.28-4.53 ; \mathrm{P}<0.01) .^{70}$ Similar results were subsequently obtained. ${ }^{71,72}$ In a multicenter, prospective, randomized study (PROLONG study), patients with abnormal D-dimer, measured one month after warfarin was stopped, were randomly assigned to either stop or resume VKAs while those with negative D-dimer did not resume anticoagulation. ${ }^{73}$ During 18 months follow-up, the incidence of recurrent VTE was $6.2 \%(4.4 \% / y)$ in those with normal Ddimer test but $2.9 \%(2.0 \% / \mathrm{y})$ and $15.0 \%(10.9 \% / \mathrm{y})$ in patients with abnormal D-dimer randomized to resume or not anticoagulation. That study demonstrated that patients with abnormal D-dimer 1 month after anticoagulation is withdrawn, have a high risk of recurrence and deserve extended anticoagulation. The predictive value for VTE recurrence has been confirmed by one systematic review ${ }^{74}$ one metaanalysis, ${ }^{75}$ and a patient-level meta-analysis. ${ }^{76}$

In the more recent, multicenter, prospective DULCIS study ${ }^{50}$ unprovoked patients underwent serial Ddimer assessment, starting during VKA treatment and several times after discontinuation. Patients with a positive D-dimer result (measured using the local routine assay and adopting specific cut-off values) $(42.3 \%$ of total 1010 patients), were recommended to continue or resume VKA anticoagulation (the only available at that time), whereas those with persistently negative results stopped anticoagulation definitively. At 18 months follow-up, primary outcomes occurred in $3.0 \% / \mathrm{y}$ of patients with negative D-dimer, and in $8.8 \% / y$ (hazard ratio 2.92, 95\% CI 1.87-9.72; $\mathrm{P}=0.0006)$ in patients who had positive $\mathrm{D}$-dimer results but refused to resume anticoagulation. The 373 patients, who resumed anticoagulation for positive Ddimer, had very few recurrences though they did have an unacceptably high incidence of major bleeding $(2.3 \% / y)$.

A higher VTE recurrence rate in patients who stopped anticoagulation for negative D-dimer testing (assessed with a qualitative assay) was recorded in a subsequent study. ${ }^{77}$ As discussed more in detail elsewhere, ${ }^{78}$ it needs to be borne in mind that the rate of positive D-dimer results in the Kearon study was much lower than expected on the basis of comparable studies, probably due to the use of an insufficiently sensitive assay. A low rate of abnormal D-dimer results translates into more false-negative results.

\section{Algorithms to predict the risk of venous thromboembolism recurrence}

Several clinical decision rules (CDR) have been proposed to help stratify patients with unprovoked VTE for the risk of recurrence so as to be able to inform the decision about duration of anticoagulation; all CDR include D-dimer measurement.

In 2008 Rodger et al. ${ }^{57}$ proposed, and recently validated ${ }^{79}$ a CDR called: Men continue and HERDOO2. According to this CDR, long-term anticoagulant treatment is always indicated for all males; while women at low risk of recurrence and who may discontinue anticoagulation are those who, after 5-7 months of oral anticoagulant therapy, have 0 or only 1 of the following features: i) post-thrombotic signs (hyperpigmentation, edema or redness in either leg); ii) D-dimer level $\geq 250 \mu \mathrm{g} / \mathrm{L}$ (measured during anticoagulation); iii) body mass index $\geq 30 \mathrm{~kg} / \mathrm{m}^{2}$; iv) age $\geq 65$ years.

Eichinger et al. ${ }^{80}$ proposed a nomogram that includes the following three variables, significantly associated with recurrence: i) sex (male $>$ female); ii) location of index VTE event (PE $>$ proximal DVT $>$ distal DVT); and iii) elevated levels of D-dimer measured after anticoagulation was stopped. The nomogram has recently been validated in different patients. ${ }^{81}$

Tosetto et al. elaborated ${ }^{82}$ and validated ${ }^{83}$ a prognostic recurrence score called $D A S H$, that includes: i) abnormal D-dimer ( 2 points); ii) age $<50$ years ( 1 point); iii) sex (male 1 point); and iv) index event in women that was associated with hormonal therapy ( -2 points). On the basis of this CDR, patients with 0 or 1 score are at low risk of recurrence and can avoid lifelong anticoagulation.

\section{The risk of bleeding during anticoagulation}

The duration of anticoagulation after VTE should balance the risk of recurrence and that of bleeding. Many factors may influence the risk of bleeding during VKA treatment, ${ }^{84}$ with complications in patients who start VKA anticoagulation for the first time significantly higher during the first 3 months of treatment than thereafter. ${ }^{85} \mathrm{~A}$ meta-analysis of available studies found a $2 \% / y$ risk of major bleeding during the first 3 months, and a $2.7 \% / y$ in the subsequent period. ${ }^{86}$ Though higher rates of bleeding can be expected in real-life treatment, phase III clinical trials on DOACs showed low rates of major bleeds (from $0.6 \% / y$ to 
$1.2 \% / y)$ in DOAC-treated patients, whereas in comparator-treated subjects (usually low-molecularweight heparin + warfarin) the rates ranged between $1.2 \% / \mathrm{y}$ and $2.2 \% / \mathrm{y} .{ }^{87}$

The ACCP AT10 ACCP guidelines say: In patients with a first VTE that is an unprovoked proximal DVT of the leg or PE and who have; a i) low or moderate bleeding risk (see text), we suggest extended anticoagulant therapy (no scheduled stop date) over 3 months of therapy (Grade 2B); and a ii) high bleeding risk (see text), we recommend 3 months of anticoagulant therapy over extended therapy (no scheduled stop date) (Grade 1B). ${ }^{20}$ It is, however, not easy to assess the individual risk of bleeding in VTE patients. The above guidelines propose a categorization of bleeding risk in anticoagulated patients on the basis of the presence of the following risk factors: age $>65$ years, age $>75$ years, previous bleeding, cancer, metastatic cancer, renal or liver failure, diabetes, previous stroke, anemia, thrombocytopenia, presence of comorbidities, antiplatelet therapy, nonsteroidal anti-inflammatory drugs, poor anticoagulant control, recent surgery, frequent falls, alcohol abuse. The risk of bleeding during anticoagulation with VKA is low in patients without risk factors, moderate in those with 1 factor, and high in those with $\geq 2$ factors. ${ }^{20}$

Other bleeding risk scores have also been proposed; it should be noticed, however, that they all have only a modest predictive value for patients with VTE. ${ }^{88}$ In conclusion, it is not easy to predict the individual risk of bleeding in VTE patients, who are generally much younger than patients treated for atrial fibrillation and usually do not have a personal history of bleeding events.

\section{The duration of treatment in the era of direct oral anticoagulants and of other antithrombotic drugs}

The DULCIS study showed that the resumption and extension of anticoagulation with VKA in patients with abnormal D-dimer was associated with a high incidence of major bleeding complications $(2.3 \% / y){ }^{50}$ Phase III trials $^{89}$ and recent real-life studies have shown that DOAC use in VTE patients is in general associated with a low rate of bleeding. It seems, therefore, reasonable to propose their use instead of VKAs for extended anticoagulation. Two randomized clinical trials have focused on the use of low-dose DOACs for extended therapy in VTE patients. A low dose of apixaban (2.5 $\mathrm{mg}$ BID) was found to be equally effective against recurrences than the standard treatment dose (5 mg BID), with a very low rate of bleeding. ${ }^{90}$ Recently, a prophylactic dose of rivaroxaban (10 $\mathrm{mg}$ OID) proved to be as effective as the standard treatment dose (20 mg OID), and more effective than aspirin $(100 \mathrm{mg} /$ day $)$, without increasing the risk of bleeding complications. ${ }^{91}$ In contrast with previous studies, ${ }^{92-94}$ these results seem to deny any advantage of using low dose aspirin for extended treatment after a VTE versus DOACs. It can be concluded that low dose apixaban or rivaroxaban may be a good opportunity for extended treatment, and that aspirin would not be a good choice, especially if we bear in mind its not negligible bleeding risk, particularly in elderly patients..$^{95}$

In line with the results of the mentioned trials on DOAC use for extended treatment, a new clinical study has been designed and is currently running: the APIDULCIS study (coordinators: Palareti G. and Prandoni P.) is an Italian, prospective, multicenter cohort study, that includes patients aged 75 years or younger, who are candidates for extended anticoagulant treatment because of a first VTE event that was unprovoked or associated with a weak risk factor. All these patients would be candidates for indefinite anticoagulation according to the ACCP guidelines; however, after completing 12 months of anticoagulant therapy (whatever the drug used), serial D-dimer testing is performed to try to distinguish subjects at low risk of recurrence (with D-dimer persistently negative) in whom extended anticoagulation can be avoided, from those (with an abnormal D-dimer result) who are at increased risk of recurrence and deserve extended anticoagulation through administration of apixaban $2.5 \mathrm{mg}$ BID for 18 months. The drug is courteously provided by Alliance BMS-Pfizer.

The effect of sulodexide administration, a glycosaminoglycan with antithrombotic and profibrinolytic actions and low bleeding risk when administered orally, has recently been investigated in a placebo-controlled, double-blind trial for prevention of recurrent VTE after a standard course of anticoagulant treatment. ${ }^{96}$ The patients who received sulodexide had a $50 \%$ reduction of recurrences versus those receiving placebo $(\mathrm{P}=0.02)$, without any occurrence of major bleeding episodes. These results are promising, especially for a potentially safe and effective protection in patients with high risk of bleeding if treated with anticoagulants (e.g. elderly patients).

\section{Practical suggestions on decision about anticoagulant duration after a venous thromboembolism event}

As shown in Table 2, a decision is easy to take for patients who have characteristics clearly justifying short or, vice-versa, indefinite anticoagulation. However, this is not true for many patients, especially those with unprovoked events, who can roughly be estimated to represent approximately half of all new VTE patients. Since the risk of bleeding does not seem to be high or is uncertain in the majority of these patients, 
most of them are candidates for indefinite anticoagulant treatment; however, we know that only $50 \%$ of them are expected to have a recurrence in 10 years without anticoagulation. ${ }^{22}$ The decision to opt for indefinite anticoagulation in all these patients is not easy. This is also recognized by the ACCP guidelines that, for a better selection of patients for extended VTE therapy, suggest including two additional criteria: patient sex (males are at higher risk of recurrence than females and abnormal D-dimer levels 1 month after anticoagulation is stopped). The ACCP guidelines also recommend involving the patients and considering their preference for the final decision. ${ }^{20}$

As practical guidance, the following procedure may be suggested to decide on duration of anticoagulant therapy after VTE:

- All patients with VTE should receive 3 to 6 months of initial anticoagulation and be re-considered after that period to assess risk of recurrence if anticoagulation is stopped and bleeding if anticoagulation is extended.

- Patients at high risk of bleeding (according to the ACCP score ${ }^{20}$ or other criteria) are informed about this risk and - in general - are advised to stop anticoagulation; all elderly patients ( $>75$ years old) are in this condition.

- All patients with leg DVT should receive compression ultrasonography (CUS) of proximal deep veins when stopping anticoagulation in order to have a baseline result in case of subsequent suspected ipsilateral recurrence. In patients with nonhigh risk of bleeding and RVT ( $>4 \mathrm{~mm}$ at CUS) a longer anticoagulation course, e.g. 6 to 12 months, may be advisable after which the persistence of RVT does not seem to have any effect on recurrence rates. ${ }^{49,50}$

- Anticoagulation should be stopped after the initial period (3 to 6 months) in patients with provoked events (associated with trigger - and removed factors).

- Patients at high risk of recurrence are advised to undergo extended anticoagulation for an indefinite time (with periodical checks to assess the balance between benefit and risks).

- Patients with an unprovoked event or associated with weak risk factors are candidates for indefinite anticoagulation, provided they are not at high risk of bleeding. They should be informed about future recurrence risk if anticoagulation is stopped and told that international guidelines recommend an indefinite anticoagulation for this condition. However, they can also be informed about the fact that an alternative management procedure is possible, ${ }^{50}$ based on use of serial D-dimer testing to assess individual risk of recurrence, and identify patients who, having a high risk (with abnormal D-dimer levels after anticoagulation is withdrawn), deserve extended anticoagulation. On the contrary, patients with persistently negative $\mathrm{D}$-dimer results are informed about their risk of recurrence is low enough not to extend anticoagulation.

- Any final decision should be taken in accordance with patient preference; after accurate and complete information is given, they may prefer extending anticoagulation for greater protection or stopping due to potential risks.

\section{References}

1. Anderson FA, Wheeler HB, Goldberg RJ, et al. A Population-based perspective of the hospital incidence and case-fatality rates of deep vein thrombosis and pulmonary embolism - the Worcester DVT study. Arch Intern Med 1991;151:933-8.

2. Nordstrom M, Lindblad B, Bergqvist D, Kjellstrom T. A prospective study of the incidence of deep-vein thrombosis within a defined urban population. J Intern Med 1992;232:155-60.

3. Oger E. Incidence of venous thromboembolism: a community-based study in western France. Thromb Haemost 2000;83:657-60.

4. Naess IA, Christiansen SC, Romundstad P, et al. Incidence and mortality of venous thrombosis: a populationbased study. J Thromb Haemost 2007;5:692-9.

5. Schulman S, Kearon C, Kakkar AK, et al. Dabigatran versus warfarin in the treatment of acute venous thromboembolism. N Engl J Med 2009;361:2342-52.

6. Buller HR. Edoxaban versus warfarin for venous thromboembolism. N Engl J Med 2014;370:80-1.

7. Bauersachs R, Berkowitz SD, Brenner B, et al. Oral rivaroxaban for symptomatic venous thromboembolism. N Engl J Med 2010;363:2499-510.

8. Agnelli G, Buller HR, Cohen A, et al. Oral apixaban for the treatment of acute venous thromboembolism. N Engl J Med 2013;369:799-808.

9. Kahn SR, Shrier I, Julian JA, et al. Determinants and time course of the postthrombotic syndrome after acute deep venous thrombosis. Ann Intern Med 2008;149:698-707.

10. Pengo V, Lensing AWA, Prins MH, et al. Incidence of chronic thromboembolic pulmonary hypertension after pulmonary embolism. N Engl J Med 2004;350:2257-64.

11. Kearon C, Hirsh J. Current concepts: management of anticoagulation before and after elective surgery. $\mathrm{N}$ Engl J Med 1997;336:1506-11.

12. Siragusa S, Cosmi B, Piovella F, Hirsh J, Ginsberg JS. Low-molecular-weight heparins and unfractionated heparin in the treatment of patients with acute venous thromboembolism: results of a meta-analysis. Am J Med 1996;100:269-77.

13. Douketis JD, Crowther MA, Foster GA, Ginsberg JS. Does the location of thrombosis determine the risk of disease recurrence in patients with proximal deep vein thrombosis? Am J Med 2001;110:515-9.

14. Heit JA, Silverstein MD, Mohr DN, et al. The epidemiology of venous thromboembolism in the community. Thromb Haemost 2001;86:452-63.

15. Boutitie F, Pinede L, Schulman S, et al. Influence of pre- 
ceding length of anticoagulant treatment and initial presentation of venous thromboembolism on risk of recurrence after stopping treatment: analysis of individual participants' data from seven trials. BMJ 2011;342: d3036.

16. Pinede L, Ninet J, Duhaut $\mathrm{P}$, et al. Comparison of 3 and 6 months of oral anticoagulant therapy after a first episode of proximal deep vein thrombosis or pulmonary embolism and comparison of 6 and 12 weeks of therapy after isolated calf deep vein thrombosis. Circulation 2001;103:2453-60.

17. Agnelli G, Prandoni P, Santamaria MG, et al. Three months versus one year of oral anticoagulant therapy for idiopathic deep venous thrombosis. N Engl J Med 2001;345:165-9.

18. Agnelli G, Prandoni P, Becattini C, et al. Extended oral anticoagulant therapy after a first episode of pulmonary embolism. Ann Intern Med 2003;139:19-25.

19. Couturaud F, Sanchez O, Pernod G, et al. Six Months vs extended oral anticoagulation after a first episode of pulmonary embolism: the PADIS-PE randomized clinical trial. JAMA 2015;314:31-40.

20. Kearon C, Akl EA, Ornelas J, et al. Antithrombotic therapy for VTE disease: CHEST guideline and expert panel report. Chest 2016;149:315-52.

21. Prandoni P, Lensing AWA, Cogo A, et al. The long-term clinical course of acute deep venous thrombosis. Ann Intern Med 1996;125:1-7.

22. Prandoni P, Noventa F, Ghirarduzzi A, et al. The risk of recurrent venous thromboembolism after discontinuing anticoagulation in patients with acute proximal deep vein thrombosis or pulmonary embolism. A prospective cohort study in 1,626 patients. Haematologica 2007;92: 199-205.

23. Heit JA, Rooke TW, Silverstein MD, et al. Trends in the incidence of venous stasis syndrome and venous ulcer: A 25-year population-based study. J Vasc Surg 2001;33: 1022-7.

24. Carrier M, Le Gal G, Wells PS, Rodger MA. Systematic review: case-fatality rates of recurrent venous thromboembolism and major bleeding events among patients treated for venous thromboembolism. Ann Intern Med 2010;152:578-89.

25. Prins MH, Lensing AWA, Prandoni P, et al. Risk of recurrent venous thromboembolism according to baseline risk factor profiles. Blood Adv 2018;2:788-96.

26. Baglin T, Douketis J, Tosetto A, et al. Does the clinical presentation and extent of venous thrombosis predict likelihood and type of recurrence? A patient-level metaanalysis. J Thromb Haemost 2010;8:2436-42.

27. Donadini MP, Dentali F, Pegoraro S, et al. Long-term recurrence of venous thromboembolism after short-term treatment of symptomatic isolated distal deep vein thrombosis: A cohort study. Vasc Med 2017;22:518-24.

28. Iorio A, Kearon C, Filippucci E, et al. Risk of recurrence after a first episode of symptomatic venous thromboembolism provoked by a transient risk factor: a systematic review. Arch Intern Med 2010;170:1710-6.

29. Schulman S, Granqvist S, Holmstrom M, et al. The duration of oral anticoagulant therapy after a second episode of venous thromboembolism. N Engl J Med 1997;336:393-8

30. Palareti G, Legnani C, Lee A, et al. A comparison of the safety and efficacy of oral anticoagulation for the treatment of venous thromboembolic disease in patients with or without malignancy. Thromb Haemost 2000;84:805-10.

31. Prandoni P, Lensing AWA, Piccioli A, et al. Recurrent venous thromboembolism and bleeding complications during anticoagulant treatment in patients with cancer and venous thrombosis. Blood 2002;100:3484-8.

32. Heit JA, Mohr DN, Silverstein MD, et al. Predictors of recurrence after deep vein thrombosis and pulmonary embolism - A population-based cohort study. Arch Intern Med 2000;160:761-8.

33. DeStefano V, Martinelli I, Mannucci PM, et al. The risk of recurrent deep venous thrombosis among heterozygous carriers of both factor V Leiden and the G20210A prothrombin mutation. N Engl J Med 1999;341:801-6.

34. Lindmarker P, Schulman S, StenLinder M, et al. The risk of recurrent venous thromboembolism in carriers and non-carriers of the G1691A allele in the coagulation factor $\mathrm{V}$ gene and the G20210A allele in the prothrombin gene. Thromb Haemost 1999;81:684-9.

35. Khamashta MA, Cuadrado MJ, Mujic F, et al. The management of thrombosis in the antiphospholipid-antibody syndrome. N Engl J Med 1995;332:993-7.

36. Eichinger S, Weltermann A, Minar E, et al. Symptomatic pulmonary embolism and the risk of recurrent venous thromboembolism. Arch Intern Med 2004;164:92-6.

37. Murin S, Romano PS, White RH. Comparison of outcomes after hospitalization for deep venous thrombosis or pulmonary embolism. Thromb Haemost 2002;88:407-14.

38. Douketis JD, Kearon C, Bates S, et al. Risk of fatal pulmonary embolism in patients with treated venous thromboembolism. JAMA 1998;279:458-62.

39. Tichelaar YI, Kluin-Nelemans HJ, Meijer K. Infections and inflammatory diseases as risk factors for venous thrombosis. A systematic review. Thromb Haemost 2012;107:827-37.

40. Piovella F, Crippa L, Barone M, et al. Normalization rates of compression ultrasonography in patients with a first episode of deep vein thrombosis of the lower limbs: association with DVT recurrence and new thrombosis. Haematologica 2002;87:515-22.

41. Prandoni P, Lensing AWA, Prins MH, et al. Residual venous thrombosis as a predictive factor of recurrent venous thromboembolism. Ann Intern Med 2002;137:955-60.

42. Galli M, Ageno W, Squizzato A, et al. Residual venous obstruction in patients with a single episode of deep vein thrombosis and in patients with recurrent deep vein thrombosis. Thromb Haemost 2005;94:93-5.

43. Siragusa S, Malato A, Saccullo G, et al. Residual vein thrombosis for assessing duration of anticoagulation after unprovoked deep vein thrombosis of the lower limbs: the extended DACUS study. Am J Hematol 2011;86:914-7.

44. Cosmi B, Legnani C, Cini M, et al. D-dimer levels in combination with residual venous obstruction and the risk of recurrence after anticoagulation withdrawal for a first idiopathic deep vein thrombosis. Thromb Haemost 2005;94:969-74.

45. Poli D, Antonucci E, Ciuti G, et al. Combination of Ddimer, F1+2 and residual vein obstruction as predictors of VTE recurrence in patients with first VTE episode after OAT withdrawal. J Thromb Haemost 2008;6:708-10.

46. Cosmi B, Legnani C, Cini M, et al. D-dimer and residual vein obstruction as risk factors for recurrence during and 
after anticoagulation withdrawal in patients with a first episode of provoked deep-vein thrombosis. Thromb Haemost 2011;105:837-45.

47. Prandoni P, Prins MH, Lensing AW, et al. Residual thrombosis on ultrasonography to guide the duration of anticoagulation in patients with deep venous thrombosis: a randomized trial. Ann Intern Med 2009;150:577-85.

48. Siragusa S, Malato A, Anastasio R, et al. Residual vein thrombosis to establish duration of anticoagulation after a first episode of deep vein thrombosis: the Duration of Anticoagulation based on Compression UltraSonography (DACUS) study. Blood 2008;112:511-5.

49. Donadini MP, Ageno W, Antonucci E, et al. Prognostic significance of residual venous obstruction in patients with treated unprovoked deep vein thrombosis: a patientlevel meta-analysis. Thromb Haemost 2014;111:172-9.

50. Palareti G, Cosmi B, Legnani C, et al. D-dimer to guide the duration of anticoagulation in patients with venous thromboembolism: a management study. Blood 2014; 124:196-203.

51. McRae S, Tran H, Schulman S, et al. Effect of patient's sex on risk of recurrent venous thromboembolism: a meta-analysis. Lancet 2006;368:371-8.

52. Roach RE, Venemans A, Cannegieter SC, Lijfering WM. Differential risks in men and women for first and recurrent venous thrombosis: the role of genes and environment: reply. J Thromb Haemost 2015;13:886-7.

53. Douketis J, Tosetto A, Marcucci M, et al. Risk of recurrence after venous thromboembolism in men and women: patient level meta-analysis. BMJ 2011;342:d813.

54. Schulman S, Lindmarker P, Holmstrom M, et al. Postthrombotic syndrome, recurrence, and death 10 years after the first episode of venous thromboembolism treated with warfarin for 6 weeks or 6 months. J Thromb Haemost 2006;4:734-42.

55. Hansson PO, Sorbo J, Eriksson H. Recurrent venous thromboembolism after deep vein thrombosis: incidence and risk factors. Arch Intern Med 2000;160:769-74.

56. Christiansen SC, Lijfering WM, Helmerhorst FM, et al. Sex difference in risk of recurrent venous thrombosis and the risk profile for a second event. J Thromb Haemost 2010;8:2159-68.

57. Rodger MA, Kahn SR, Wells PS, et al. Identifying unprovoked thromboembolism patients at low risk for recurrence who can discontinue anticoagulant therapy. CMAJ 2008;179:417-26.

58. Eichinger S, Hron G, Bialonczyk C, et al. Overweight, obesity, and the risk of recurrent venous thromboembolism. Arch Intern Med 2008;168:1678-83.

59. Kyrle PA, Minar E, Hirschl M, et al. High plasma levels of factor VIII and the risk of recurrent venous thromboembolism. N Engl J Med 2000;343:457-62.

60. Legnani C, Cosmi B, Cini M, et al. High plasma levels of factor VIII and risk of recurrence of venous thromboembolism. Br J Haematol 2004;124:504-10.

61. Weltermann A, Eichinger S, Bialonczyk C, et al. The risk of recurrent venous thromboembolism among patients with high factor IX levels. J Thromb Haemost 2003;1:28-32.

62. DeStefano V, Martinelli I, Mannucci PM, et al. The risk of recurrent venous thromboembolism among heterozygous carriers of the G20210A prothrombin gene mutation. Br J Haematol 2001;113:630-5.
63. Baglin T, Luddington R, Brown K, Baglin C. Incidence of recurrent venous thromboembolism in relation to clinical and thrombophilic risk factors: prospective cohort study. Lancet 2003;362:523-6.

64. Ho WK, Hankey GJ, Quinlan DJ, Eikelboom JW. Risk of recurrent venous thromboembolism in patients with common thrombophilia: a systematic review. Arch Intern Med 2006;166:729-36.

65. Watson HG, Baglin TP. Guidelines on travel-related venous thrombosis. Br J Haematol 2011;152:31-4.

66. Novacek G, Weltermann A, Sobala A, et al. Inflammatory bowel disease is a risk factor for recurrent venous thromboembolism. Gastroenterology 2010;139:779-87.

67. Heit JA, Ofallon WM, Petterson TM, et al. Relative impact of risk factors for deep vein thrombosis and pulmonary embolism - A population-based study. Arch Intern Med 2002;162:1245-8.

68. Ageno W, Agnelli G, Imberti D, et al. Prevalence of risk factors for venous thromboembolism in the Italian population: results of a cross-sectional study from the Master Registry. Intern Emerg Med 2013;8:575-80.

69. Ageno W, Squizzato A, Dentali F. Should the commonly accepted definition of unprovoked venous thrombembolism be revisited? Thromb Haemost 2012; 107:806-7.

70. Palareti G, Legnani C, Cosmi B, et al. Risk of venous thromboembolism recurrence: High negative predictive value of D-dimer performed after oral anticoagulation is stopped. Thromb Haemost 2002;87:7-12.

71. Eichinger S, Minar E, Bialonczyk C, et al. D-dimer levels and risk of recurrent venous thromboembolism. JAMA Journal of the American Medical Association 2003;290:1071-4.

72. Shrivastava S, Ridker PM, Glynn RJ, et al. D-dimer, factor VIII coagulant activity, low-intensity warfarin and the risk of recurrent venous thromboembolism. J Thromb Haemost 2006;4:1208-14.

73. Palareti G, Cosmi B, Legnani C, et al. D-dimer testing to determine the duration of anticoagulation therapy. $\mathrm{N}$ Engl J Med 2006;355:1780-9.

74. Verhovsek M, Douketis JD, Yi Q, et al. Systematic review: D-dimer to predict recurrent disease after stopping anticoagulant therapy for unprovoked venous thromboembolism. Ann Intern Med 2008;149:481-90, W94.

75. Bruinstroop E, Klok FA, Van De Ree MA, et al. Elevated $\mathrm{D}$-dimer levels predict recurrence in patients with idiopathic venous thromboembolism: a meta-analysis. J Thromb Haemost 2009;7:611-8.

76. Douketis J, Tosetto A, Marcucci M, et al. Patient-level meta-analysis: effect of measurement timing, threshold, and patient age on ability of D-dimer testing to assess recurrence risk after unprovoked venous thromboembolism. Ann Intern Med 2010;153:523-31.

77. Kearon C, Spencer FA, O'Keeffe D, et al. D-dimer testing to select patients with a first unprovoked venous thromboembolism who can stop anticoagulant therapy: a cohort study. Ann Intern Med 2015;162:27-34.

78. Palareti G. d-Dimer Testing in patients with a first unprovoked venous thromboembolism. Ann Intern Med 2015;162:670-1.

79. Rodger MA, Le Gal G, Anderson DR, et al. Validating the HERDOO2 rule to guide treatment duration for women with unprovoked venous thrombosis: multina- 
tional prospective cohort management study. BMJ 2017;356:j1065.

80. Eichinger S, Heinze G, Jandeck LM, Kyrle PA. Risk assessment of recurrence in patients with unprovoked deep vein thrombosis or pulmonary embolism: the Vienna prediction model. Circulation 2010;121:1630-6.

81. Marcucci M, Iorio A, Douketis JD, et al. Risk of recurrence after a first unprovoked venous thromboembolism: external validation of the Vienna Prediction Model with pooled individual patient data. J Thromb Haemost 2015;13:775-81.

82. Tosetto A, Iorio A, Marcucci M, et al. Predicting disease recurrence in patients with previous unprovoked venous thromboembolism: a proposed prediction score (DASH). J Thromb Haemost 2012;10:1019-25.

83. Tosetto A, Testa S, Martinelli I, et al. External validation of the DASH prediction rule: a retrospective cohort study. J Thromb Haemost 2017;15:1963-70.

84. Palareti G, Cosmi B. Bleeding with anticoagulation therapy - who is at risk, and how best to identify such patients. Thromb Haemost 2009; 102:268-78.

85. Palareti G, Leali N, Coccheri S, et al. Bleeding complications of oral anticoagulant treatment: an inception- cohort, prospective collaborative study (ISCOAT). Italian Study on Complications of Oral Anticoagulant Therapy. Lancet 1996;348:423-8.

86. Linkins LA, Choi PT, Douketis JD. Clinical impact of bleeding in patients taking oral anticoagulant therapy for venous thromboembolism: a meta-analysis. Ann Intern Med 2003;139:893-900.

87. Palareti G. Direct oral anticoagulants and bleeding risk (in comparison to vitamin $\mathrm{K}$ antagonists and heparins), and the treatment of bleeding. Semin Hematol 2014;51: 102-11.

88. Riva N, Bellesini M, Di Minno MN, et al. Poor predic- tive value of contemporary bleeding risk scores during long-term treatment of venous thromboembolism. A multicentre retrospective cohort study. Thromb Haemost 2014;112:511-21.

89. van Es N, Coppens M, Schulman S, et al. Direct oral anticoagulants compared with vitamin $\mathrm{K}$ antagonists for acute venous thromboembolism: evidence from phase 3 trials. Blood 2014;124:1968-75.

90. Agnelli G, Buller HR, Cohen A, et al. Apixaban for extended treatment of venous thromboembolism. N Engl J Med 2013;368:699-708.

91. Weitz JI, Lensing AWA, Prins MH, et al. Rivaroxaban or aspirin for extended treatment of venous thromboembolism. N Engl J Med 2017;376:1211-22.

92. Becattini C, Agnelli G, Schenone A, et al. Aspirin for preventing the recurrence of venous thromboembolism. N Engl J Med 2012;366:1959-67.

93. Brighton TA, Eikelboom JW, Mann K, et al. Low-dose aspirin for preventing recurrent venous thromboembolism. N Engl J Med 2012;367:1979-87.

94. Simes J, Becattini C, Agnelli G, et al. Aspirin for the prevention of recurrent venous thromboembolism: the INSPIRE collaboration. Circulation 2014;130:1062-71.

95. Mant J, Hobbs FDR, Fletcher K, et al. Warfarin versus aspirin for stroke prevention in an elderly community population with atrial fibrillation (The Birmingham Atrial Fibrillation Treatment of the Aged Study, BAFTA): a randomised controlled trial. Lancet 2007; 370:493-503.

96. Andreozzi GM, Bignamini AA, Davi G, et al. Sulodexide for the prevention of recurrent venous thromboembolism: the sulodexide in secondary prevention of recurrent deep vein thrombosis (SURVET) study: a multicenter, randomized, double-blind, placebo-controlled trial. Circulation 2015;132:1891-7. 